\title{
Positive phase space transformation incompatible with classical physics
}

\author{
Wonmin Son, ${ }^{1,2,3}$ Johannes Kofler, ${ }^{4,5}$ M. S. Kim, ${ }^{6}$ Vlatko Vedral, ${ }^{1,2,3}$ and Časlav Brukner ${ }^{4,5}$ \\ ${ }^{I}$ The School of Physics and Astronomy, University of Leeds, LS2 9JT Leeds, United Kingdom \\ ${ }^{2}$ Centre for Quantum Technologies, National University of Singapore, 3 Science Drive 2, Singapore 117543, Singapore \\ ${ }^{3}$ Department of Physics, National University of Singapore, 2 Science Drive 3, Singapore 117543, Singapore \\ ${ }^{4}$ Fakultät für Physik, Universität Wien, Boltzmanngasse 5, A-1090 Wien, Austria \\ ${ }^{5}$ Institut für Quantenoptik und Quanteninformation, Österreichische Akademie der Wissenschaften, Boltzmanngasse 3, A-1090 Wien, Austria \\ ${ }^{6}$ School of Mathematics and Physics, The Queen's University, Belfast BT7 1NN, United Kingdom
}

(Dated: November 5, 2018)

\begin{abstract}
Bell conjectured that a positive Wigner function does not allow violation of the inequalities imposed by local hidden variable theories. A requirement for this conjecture is "when phase space measurements are performed". We introduce the theory-independent concept of "operationally local transformations" which refers to the change of the switch on a local measurement apparatus. We show that two separated parties, performing only phase space measurements on a composite quantum system with a positive Wigner function and performing only operationally local transformations that preserve this positivity, can nonetheless violate Bell's inequality. Such operationally local transformations are realized using entangled ancillae.
\end{abstract}

Quantum theory makes only probabilistic predictions in the description of nature. Since the seminal gedanken experiment by Einstein, Podolsky and Rosen (EPR) [1], there had been a long debate whether one can go beyond probabilities and arrive at a deterministic description of nature. Bell has shown that theories which base on the two assumptions of realism and locality have to fulfill certain inequalities [2]. Realism assumes the existence of "hidden variables" which determine the properties of physical systems prior to and independent of measurements. Locality assumes that these properties cannot be influenced by space-like separated events. Bell's inequalities are violated by quantum mechanical predictions for entangled states, and under reasonable assumptions numerous experiments have disproved local realism. Entanglement also plays a decisive role in the new field of quantum information, where quantum correlations are exploited to perform classically impossible tasks (e.g., quantum cryptography and quantum communication complexity [3]).

To construct a local hidden variable model for correlation measurements on two particles, it is necessary to find a positive probability distribution over their local hidden variables. One might think that such a model is constructed by finding a representation of the state in terms of a positive probability distribution of phase space variables (position and momentum). However, it has been argued that the existence of such a distribution is not a sufficient condition for satisfying Bell's inequality [4]. Rather, one has to also restrict oneself to certain measurement observables (phase space measurements).

We will explain the necessary conditions for the existence of a local hidden variable model using the Wigner quasi probability distribution (which can be negative in general) and the historic example of the (non-normalized) entangled EPR state $\int_{-\infty}^{\infty} \mathrm{d} x|x\rangle_{1}\left|x+x_{0}\right\rangle_{2}=\int_{-\infty}^{\infty} \mathrm{d} p \exp \left(\frac{\mathrm{i}}{\hbar} x_{0} p\right)|p\rangle_{1}|-p\rangle_{2}$. Here, $x$ and $p$ denote position and momentum, $x_{0}$ is a constant, and indices label the two particles. Bell conjectured that this state does not violate Bell's inequality imposed by local hidden variables due to the fact that-although the state is entangled (non-separable)-its Wigner function [5] $W\left(x_{1}, p_{1}, x_{2}, p_{2}\right)=$ $\delta\left(x_{1}-x_{2}+x_{0}\right) \delta\left(p_{1}+p_{2}\right)$ is positive in the whole phase space [2]. Here, $x_{1}, p_{1}, x_{2}, p_{2}$ can serve as local hidden variables with $W$ being a positive probability distribution, and correlations can be computed via suitable integration over the Wigner function. Accordingly, no Bell inequality can be violated. The EPR states can be experimentally realized e.g. via two-mode squeezed states [6].

While Bell's conjecture is true for phase space measurements, it has been shown that nevertheless there are certain other measurements for which local realism can be violated although the state has a positive Wigner function [4, 7, 8]. (One example is the parity measurement of the number of photons in the two-mode squeezed state.) The reason is that "[t]he Wigner representations of quantum observables cannot be in general interpreted as phase space distributions of possible experimental outcomes. [...] This enables violation of Bell's inequalities even for quantum states described by positivedefinite Wigner functions." [4]

Consider the expectation value of correlations of outcomes $a$ and $b$ for given local measurements,

$$
E=\sum a b p(a, b)
$$

where $p(a, b)$ is the joint probability for the respective outcomes. This correlation can in general be obtained by

$$
E=\int \mathrm{d} x_{1} \mathrm{~d} p_{1} \mathrm{~d} x_{2} \mathrm{~d} p_{2} A\left(x_{1}, p_{1}\right) B\left(x_{2}, p_{2}\right) W\left(x_{1}, p_{1}, x_{2}, p_{2}\right)
$$

where $A\left(x_{1}, p_{1}\right)$ and $B\left(x_{2}, p_{2}\right)$ are the Wigner representations of the measurement observables. From the general framework introduced in [9], it follows that, in case of positive $W$ and bounded $A\left(x_{1}, p_{1}\right), B\left(x_{2}, p_{2}\right)$, the corresponding correlations admit a local realistic description and, therefore, satisfy any correlation Bell inequality (possibly rescaled) for any numbers of settings and outcomes at each site. The situation is, however, quite different whenever the functions $A\left(x_{1}, p_{1}\right)$, $B\left(x_{2}, p_{2}\right)$ are unbounded. For example, in Ref. [4], the parity measurement outcomes $a, b$ can have only values +1 or 
-1 , but the Wigner representations $A\left(x_{1}, p_{1}\right), B\left(x_{2}, p_{2}\right)$ are given by delta functions. The latter makes the Bell inequality void [4] even in the presence of positive $W$.

It is still questioning whether the positivity of the Wigner function and using phase space observables are sufficient ingredients to claim whether the system is genuinely classical. This is an important question since it might be related to the recent dispute on the power of nuclear magnetic resonance quantum computation. It is claimed that a system in a highly noisy statistical mixture can be used as a resource for quantum computation [10]. Although all states involved in the experiment have a local realistic model, one can exploit the fact that the transformations between the states are essentially quantum mechanical, not having any classical description [11]. This implies that the describability of a prepared system in terms of local realism may not rule out its usefulness as a resource for quantum information processing.

Throughout the paper, we will use the term "local transformation" in an operational meaning. It is defined theoryindependently and refers to the change of a switch on a local measurement apparatus. A general measurement at one observer side can be understood as a concatenation of a corresponding (operationally) local state transformation and a fixed measurement (in the computational basis). Naively, one would assume that if one has a local hidden variable model for a quantum state and one applies only operationally local transformations, no Bell inequalities can be violated. We will show that this is wrong.

We present an explicit example of a quantum state with a positive Wigner function and operationally local transformations that keep this positivity, as well as a fixed phase space measurement on each side, which nevertheless allows to violate Bell's inequality. This demonstrates that even the conjunction of (i) positivity of the system's Wigner function, (ii) fixed phase space measurements, and (iii) operationally local transformations preserving the positivity of the system's Wigner function is not sufficient to claim the possibility of a local realistic model. The trick here is that although the quantum state itself has a local hidden variable model, the used operationally local transformations are completely positive (CP) maps which can only be simulated in the hidden variable model via non-local transformations. Such a situation is achieved by exploiting additional entangled particles (called "ancillae" in quantum information theory).

We will now derive a Bell inequality in terms of local phase space transformations in local realistic theories, and our results can therefore be understood as a "Bell theorem for local transformations". These theories are based on two postulates: (I) Realism: Systems have well-defined properties prior to and independent of measurement. (II) Locality: The properties on one side are independent of any (space-like) separated events-in particular of operationally local transformationson the other side.

Consider two separated observers, usually called Alice and Bob, who measure the phase space observables $A_{i}$ and $B_{j}$ $(i, j=1,2)$ of a system with positive Wigner function $W$. The expectation value of the correlation reads

$$
\left\langle A_{i} B_{j}\right\rangle_{W}=\int \mathrm{d} \mathbf{z}_{1} \mathrm{~d} \mathbf{z}_{2} W\left(\mathbf{z}_{1}, \mathbf{z}_{2}\right) A_{i}\left(\mathbf{z}_{1}\right) B_{j}\left(\mathbf{z}_{2}\right),
$$

with $\mathbf{z}_{1}=\left(x_{1}, p_{1}\right)$ and $\mathbf{z}_{2}=\left(x_{2}, p_{2}\right)$ the shortcuts for the local hidden variables (e.g. position and momentum) of Alice and Bob, respectively. Note that in local phase space measurements the outcomes depend only on the position and momentum of the local particles. Eq. (1) represents a local realistic model for phase space measurements on a bipartite quantum state with a positive Wigner function, or the classical average of products under phase space measurements on two classical particles. Taking dichotomic observables $A_{i}$ and $B_{j}$, the Clauser-Horne-Shimony-Holt (CHSH) inequality [12] reads

$$
\left\langle A_{1} B_{1}\right\rangle_{W}+\left\langle A_{1} B_{2}\right\rangle_{W}+\left\langle A_{2} B_{1}\right\rangle_{W}-\left\langle A_{2} B_{2}\right\rangle_{W} \leq 2 .
$$

The correlation $\left\langle A_{i} B_{j}\right\rangle_{W}$ can also be established by performing operationally local transformations, which-within local realistic theories - have the form

$$
\mathbf{z}_{1} \rightarrow \mathbf{z}_{1}^{(i)}=\mathbf{S}_{i}\left(\mathbf{z}_{1}\right), \quad \mathbf{z}_{2} \rightarrow \mathbf{z}_{2}^{(j)}=\mathbf{T}_{j}\left(\mathbf{z}_{2}\right),
$$

followed by measuring fixed phase space observables (no in$\operatorname{dex}) A\left(\mathbf{z}_{1}^{(i)}\right)=A_{i}\left(\mathbf{S}_{i}^{-1}\left(\mathbf{z}_{1}^{(i)}\right)\right), B\left(\mathbf{z}_{2}^{(j)}\right)=B_{j}\left(\mathbf{T}_{j}^{-1}\left(\mathbf{z}_{2}^{(j)}\right)\right)$ :

$$
\langle A B\rangle_{W_{i j}}=\int \mathrm{d} \mathbf{z}_{1}^{(i)} \mathrm{d} \mathbf{z}_{2}^{(j)} J_{i j} W_{i j}\left(\mathbf{z}_{1}^{(i)}, \mathbf{z}_{2}^{(j)}\right) A\left(\mathbf{z}_{1}^{(i)}\right) B\left(\mathbf{z}_{2}^{(j)}\right) .
$$

In eq. (4) $W_{i j}\left(\mathbf{z}_{1}^{(i)}, \mathbf{z}_{2}^{(j)}\right)=W\left(\mathbf{S}_{i}^{-1}\left(\mathbf{z}_{1}^{(i)}\right), \mathbf{T}_{j}^{-1}\left(\mathbf{z}_{2}^{(j)}\right)\right)$ is the Wigner function of the state after the invertible local transformations $\mathbf{S}_{i}$ and $\mathbf{T}_{j}$, and $J_{i j}$ is the determinant of the Jacobian of this transformation. The equivalence of eqs. (1) and (4) is closely related to the equivalence of active and passive transformations. Here it is crucial that within local hidden variable models the operationally local transformations (3) are local transformations of the hidden variables, i.e. $\mathbf{z}_{1}^{(i)}=\mathbf{S}_{i}\left(\mathbf{z}_{1}\right)$ is not a function of $\mathbf{z}_{2}$ and $\mathbf{z}_{2}^{(j)}=\mathbf{T}_{j}\left(\mathbf{z}_{2}\right)$ is not a function of $\mathbf{z}_{1}$.

Taking again dichotomic observables $A_{i}$ and $B_{j}$, allows to rewrite the $\mathrm{CHSH}$ inequality in the form

$$
\langle A B\rangle_{W_{11}}+\langle A B\rangle_{W_{12}}+\langle A B\rangle_{W_{21}}-\langle A B\rangle_{W_{22}} \leq 2 .
$$

We will show that four positive Wigner functions allow to violate the $\mathrm{CHSH}$ inequality for fixed phase space quantum measurements, even though they remain positive throughout the transformations. Note that this is different from choosing four positive arbitrary Wigner functions $f_{i j}$ for which the above inequality with the terms $\langle A B\rangle_{f_{i j}}$ can always be violated. The point is that the functions $W_{i j}$ in (5) are obtained by operationally local transformations.

Consider the mixture of coherent states

$$
\hat{\rho}_{0}=\frac{1}{2}\left(|\alpha, \alpha\rangle_{12}\langle\alpha, \alpha|+|-\alpha,-\alpha\rangle_{12}\langle-\alpha,-\alpha|\right),
$$

where $|\alpha\rangle$ denotes a coherent state of complex amplitude $\alpha$ [13] and 1 and 2 label Alice and Bob's particle respectively. 
For large $|\alpha|$, the two states $|\alpha\rangle$ and $|-\alpha\rangle$ become almost orthogonal as $|\langle\alpha \mid-\alpha\rangle|^{2}=e^{-4|\alpha|^{2}} \approx 0$. From now on, we assume that $|\alpha|$ is sufficiently large.

The Wigner function of the state $\hat{\rho}_{0}, W_{\hat{\rho}_{0}}\left(\beta_{1}, \beta_{2}\right)=$ $\frac{1}{2}\left[W_{\alpha}\left(\beta_{1}\right) W_{\alpha}\left(\beta_{2}\right)+W_{-\alpha}\left(\beta_{1}\right) W_{-\alpha}\left(\beta_{2}\right)\right]$, is positive at every point in phase space because the Wigner function for a single coherent state $|\alpha\rangle$, i.e. $W_{\alpha}(\beta)=\frac{2}{\pi} e^{-2|\alpha-\beta|^{2}}$, is positive. Here, the complex numbers $\beta_{1}$ and $\beta_{2}$ take the role of Alice's and Bob's local hidden variables (as $\mathbf{z}_{1}$ and $\mathbf{z}_{2}$ in eq. (11). Note that all quasi probability distributions for the separable state (6) are positive, and therefore the results of this work are representation-independent.

In a Bell experiment, Alice and Bob measure the local quadratures (as a phase space measurement) and assign either the value +1 or -1 depending on the sign of the quadrature. Thus, Alice' observable is $\hat{A}(\theta) \equiv \operatorname{Sign}\left[\hat{x}_{\theta}\right]$ where $\hat{x}_{\theta}=\cos \theta \hat{x}+\sin \theta \hat{p}$ is the quadrature operator (along the angle $\theta$ ) with $\hat{x}$ and $\hat{p}$ the position and momentum operator [14]. The explicit form of the measurement operator is $\hat{A}(\theta)=\left(\int_{0}^{\infty} \mathrm{d} x_{\theta}-\int_{-\infty}^{0} \mathrm{~d} x_{\theta}\right)\left|x_{\theta}\right\rangle\left\langle x_{\theta}\right|$ where $\left|x_{\theta}\right\rangle$ is eigenstate of the quadrature operator $\hat{x}_{\theta}$. We assume that Bob measures his system with the same type of measurement $\hat{B}(\varphi)$. The correlation between Alice and Bob takes the form $\left\langle A_{i} B_{j}\right\rangle=$ $\operatorname{Tr}\left[\hat{A}\left(\theta_{i}\right) \hat{B}\left(\varphi_{j}\right) \hat{\rho}_{0}\right]$ which can be used for the CHSH inequality.

Now we will show that one can violate the CHSH inequality under operationally local transformations. The initial state (6) can be transformed into the state

$$
\hat{\rho}\left(\theta_{i}, \varphi_{j}\right)=\cos ^{2}\left(\theta_{i}-\varphi_{j}\right) \hat{\rho}_{0}+\sin ^{2}\left(\theta_{i}-\varphi_{j}\right) \hat{\rho}_{1}
$$

with $\hat{\rho}_{1} \equiv \frac{1}{2}(|\alpha,-\alpha\rangle\langle\alpha,-\alpha|+|-\alpha, \alpha\rangle\langle-\alpha, \alpha|)$, and where $\theta_{i}$ and $\varphi_{j}$ denote local parameters for the operationally local transformations and are controlled by Alice and Bob, respectively. It is not immediately obvious how this state can be generated but we will discuss this later. It is straightforward that the positivity of the Wigner function of the transformed state $\hat{\rho}\left(\theta_{i}, \varphi_{j}\right)$ is preserved since $W_{i j}\left(\beta_{1}, \beta_{2}\right)=$ $\frac{1}{2}\left\{\cos ^{2}\left(\theta_{i}-\varphi_{j}\right)\left[W_{\alpha}\left(\beta_{1}\right) W_{\alpha}\left(\beta_{2}\right)+W_{-\alpha}\left(\beta_{1}\right) W_{-\alpha}\left(\beta_{2}\right)\right]+\sin ^{2}\left(\theta_{i}-\right.\right.$ $\left.\left.\varphi_{j}\right)\left[W_{\alpha}\left(\beta_{1}\right) W_{-\alpha}\left(\beta_{2}\right)+W_{-\alpha}\left(\beta_{1}\right) W_{\alpha}\left(\beta_{2}\right)\right]\right\}$ which has only positive contributions. As under the transformations the state remains separable and also with positive Wigner function, one could naively characterize this transformation as classical.

Contrary to this intuition, we will show that $W_{11}, W_{12}$, $W_{21}$, and $W_{22}$ allow to violate the CHSH inequality (5). The correlation function for the state (7) with fixed ("position left/right") phase space measurements $\hat{A} \equiv \hat{A}(0)$ and $\hat{B} \equiv \hat{B}(0)$ is $\langle A B\rangle_{W_{i j}}=\operatorname{Tr}\left[\hat{A} \hat{B} \hat{\rho}\left(\theta_{i}, \varphi_{j}\right)\right]=\cos \left[2\left(\theta_{i}-\right.\right.$ $\left.\left.\varphi_{j}\right)\right] \operatorname{Erf}^{2}\left(\alpha_{r}\right)$ with $\alpha_{r} \equiv \operatorname{Re}(\alpha)$. It is notable that the correlation function cannot be factorized into individual parts for Alice and Bob. The CHSH expression $C\left(\theta_{1}, \theta_{2}, \varphi_{1}, \varphi_{2}\right) \equiv$ $\langle A B\rangle_{11}+\langle A B\rangle_{12}+\langle A B\rangle_{21}-\langle A B\rangle_{22}$ becomes $C\left(\theta_{1}, \theta_{2}, \varphi_{1}, \varphi_{2}\right)=$ $\left(\Delta_{11}+\Delta_{12}+\Delta_{21}-\Delta_{22}\right) \operatorname{Erf}^{2}\left(\alpha_{r}\right)$ where $\Delta_{i j} \equiv \cos \left[2\left(\theta_{i}-\right.\right.$ $\left.\varphi_{j}\right)$ ]. With a proper choice of the local parameters, e.g. $\left(\theta_{1}, \theta_{2}, \varphi_{1}, \varphi_{2}\right)=(0, \pi / 4, \pi / 8,-\pi / 8)$, the CHSH expression $C(0, \pi / 4, \pi / 8,-\pi / 8)=2 \sqrt{2} \operatorname{Erf}^{2}\left(\alpha_{r}\right)$ violates the CHSH inequality when $\alpha_{r}>0.9957$. Thus, we can conclude that operationally local transformations applied on a quantum state with a positive Wigner function can violate a Bell inequality, even though they keep the positivity of the Wigner function. To simulate the violation, at least one of the transformations between the four Wigner functions modeled by the hidden variables must be non-local, i.e. cannot be written as in eqs. (3) but only as $\mathbf{z}_{1}^{(i)}=\mathbf{S}_{i}\left(\mathbf{z}_{1}, \mathbf{z}_{2}\right), \mathbf{z}_{2}^{(j)}=\mathbf{T}_{j}\left(\mathbf{z}_{1}, \mathbf{z}_{2}\right)$.

We demonstrate that it is not possible to realize the set of transformations leading to a violation by local quantum operations (allowing classically correlated ancillae at both sides). Let us first consider the case where only Alice performs a general operation on her particle and Bob does nothing on his particle. Then, the transformation from (6) to (7) can be described by a positive operator-sum representation at Alice's side $\hat{\mathcal{L}}_{i}^{(A)}\left(\hat{\rho}_{0}\right)=\sum_{\mu=1}^{2} \hat{\mathcal{E}}_{\mu}^{i} \hat{\rho}_{0} \hat{\mathcal{E}}_{\mu}^{i \dagger}$ where $\hat{\rho}_{0}$ is the initial state in (6). Since the transformation changes only the diagonal elements, the most general positive operators for the local transformation by Alice are $\hat{\mathcal{E}}_{1}^{i}=\cos \theta_{i}(|\alpha\rangle\langle\alpha|+|-\alpha\rangle\langle-\alpha|)$ and $\hat{\mathcal{E}}_{2}^{i}=$ $\sin \theta_{i}(|\alpha\rangle\langle-\alpha|+|-\alpha\rangle\langle\alpha|)$ which describe the bit flip channel of the qubit and satisfy $\sum_{\mu=1}^{2} \hat{\mathcal{E}}_{\mu}^{i \dagger} \hat{\mathcal{E}}_{\mu}^{i}=\mathbb{1}$. Neither the phase flip nor the bit-phase flip operation can produce the transformation as they would introduce non-diagonal elements [3]. After the operation, the state becomes $\mathcal{L}_{i}^{(A)}\left(\hat{\rho}_{0}\right)=\hat{\rho}\left(\theta_{i}, 0\right)$. If Bob performs the same local operation on his particle with $\varphi_{j}$ instead of $\theta_{i}$, then the state is transformed into $\hat{\mathcal{L}}_{j}^{(B)}\left(\hat{\rho}_{0}\right)=\rho\left(0, \varphi_{j}\right)$. However, the composition of the local operations by Alice and Bob does not transform the initial state into (7), i.e.

$$
\hat{\mathcal{L}}_{i}^{(A)} \hat{\mathcal{L}}_{j}^{(B)}\left(\hat{\rho}_{0}\right)=\sum_{\mu, v=1}^{2}\left(\hat{\mathcal{E}}_{\mu}^{i} \otimes \hat{\mathcal{E}}_{v}^{j}\right) \hat{\rho}_{0}\left(\hat{\mathcal{E}}_{\mu}^{i \dagger} \otimes \hat{\mathcal{E}}_{v}^{j \dagger}\right) \neq \hat{\rho}\left(\theta_{i}, \varphi_{j}\right)
$$

for arbitrary local parameters $\theta_{i}$ and $\varphi_{j}$. This shows that the initial state $\hat{\rho}_{0}$, eq. (6), cannot be transformed into the state $\hat{\rho}\left(\theta_{i}, \varphi_{j}\right)$, eq. (7), by any local quantum operations possibly assisted by classically correlated ancillae.

We now show how the state transformation can be obtained by local quantum operations assisted by entangled ancillae. Note that such a quantum operation is an operationally local transformation. Let us assume that in addition to the initial state $\hat{\rho}_{0}$ Alice and Bob share an entangled state $|\psi\rangle_{34}=$ $\frac{1}{\sqrt{2}}\left(|00\rangle_{34}+|11\rangle_{34}\right)$ where the subscripts 3 and 4 denote the qubit ancillae belonging to Alice and Bob, respectively. Then, Alice rotates her original particle (label 1) together with her ancilla (label 3 ) by the following local unitary operation

$$
\hat{U}_{13}\left(\theta_{i}\right)=\left(\begin{array}{cccc}
\cos \theta_{i} & -\sin \theta_{i} & 0 & 0 \\
0 & 0 & \sin \theta_{i} & \cos \theta_{i} \\
0 & 0 & \cos \theta_{i} & -\sin \theta_{i} \\
\sin \theta_{i} & \cos \theta_{i} & 0 & 0
\end{array}\right)
$$

where the matrix basis is $\left\{|\alpha, 0\rangle_{13},|\alpha, 1\rangle_{13},|-\alpha, 0\rangle_{13}\right.$, $\left.|-\alpha, 1\rangle_{13}\right\}$. Bob applies the similar unitary $\hat{U}_{24}\left(\varphi_{j}\right)$ to his particle (label 2) and ancilla (label 4). Then the initial state $\hat{\rho}_{0}$ is transformed to

$$
\operatorname{Tr}_{34}\left[\hat{U}_{13}\left(\theta_{i}\right) \hat{U}_{24}\left(\varphi_{j}\right) \hat{\rho}_{0}|\psi\rangle_{34}\langle\psi| \hat{U}_{13}^{\dagger}\left(\theta_{i}\right) \hat{U}_{24}^{\dagger}\left(\varphi_{j}\right)\right]=\hat{\rho}\left(\theta_{i}, \varphi_{j}\right)
$$



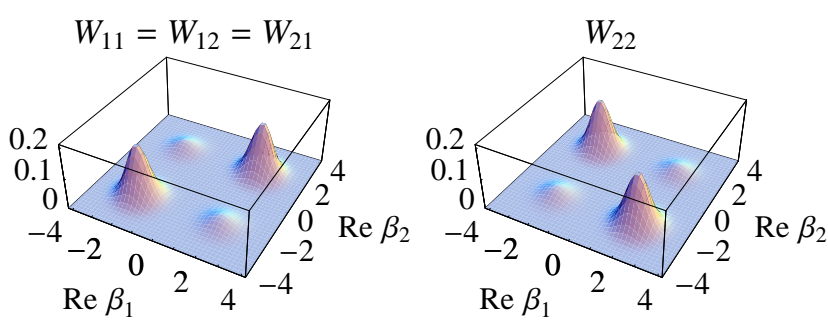

FIG. 1: (Color online.) Wigner functions $W_{i j}$ of the density matrix $\hat{\rho}\left(\theta_{i}, \varphi_{j}\right)$, eq. (7), for the transformations $\left(\theta_{i}, \varphi_{j}\right)$ with $i, j=1,2$ and $\left(\theta_{1}, \theta_{2}, \varphi_{1}, \varphi_{2}\right)=(0, \pi / 4, \pi / 8,-\pi / 8)$. The $W_{i j}$ are drawn for $\alpha=2$ as a function of the real parts of Alice's and Bob's hidden variables $\beta_{1}$ and $\beta_{2}$, respectively.

where $\operatorname{Tr}_{34}$ denotes the partial trace over the ancillae. (In general, operationally local transformations are represented in quantum mechanics as in eq. (9) using CP-maps instead of unitaries as well as general initial states of the system and the ancillae.) Thus the required transformation and the violation of the CHSH inequality can be achieved via operationally local transformations only if they involve entangled ancillae. Note that the measurements are performed on the particles 1 and 2 only and not the ancillae.

In Fig. 1] the Wigner functions $W_{i j}\left(\beta_{1}, \beta_{2}\right)$ for the state (7) are plotted (as a function of the real parts of $\beta_{1}, \beta_{2}$ ), for the transformations $\left(\theta_{i}, \varphi_{j}\right)$ with $i, j=1,2$ and $\left(\theta_{1}, \theta_{2}, \varphi_{1}, \varphi_{2}\right)=$ $(0, \pi / 4, \pi / 8,-\pi / 8)$. We chose $|\alpha|=2$ which corresponds to an overlap $|\langle\alpha \mid-\alpha\rangle|^{2} \approx 10^{-7}$. All four Wigner functions are positive and thus individually allow a local realistic description of phase space measurements. Obvious local transformations of the hidden variables $\left(\beta_{1}, \beta_{2}\right)$ that bring $W_{11}$ to $W_{12}$ and $W_{11}$ to $W_{21}$ are identities. Since then, local realistically, the transformation from $W_{11}$ to $W_{22}$ has to be the identity as well, all four Wigner functions would have to be the same. However, quantum mechanically, $W_{22}$ is different from the other functions. The violation of the CHSH inequality (5) for the position left/right measurement shows that no local transformations of the hidden variables exist that can reproduce all four Wigner functions.

It is usually assumed that no violation of Bell's inequality is possible for composite systems with positive Wigner function and phase space measurements. This is because the Wigner function itself can serve as a probability distribution of local hidden variables. Here we showed that this statement should be taken with care. Implementing transformations that keep the positivity of the Wigner function of a composite system at all times, we have demonstrated that a Bell inequality can be violated performing phase space measurements. This is possible by exploiting entangled ancillae for the transformations and the total Wigner function of the composite system and the ancillae is negative. The important point is that the transformations - even if they are assisted with entangled ancillae-are operationally local in the sense that they are implemented in a laboratory by a switch on a local measurement apparatus. If one wants to model such operationally local transformations in a hidden variable model, one would require non-local transformations of the hidden variables.

We thank J. Dunningham for discussions and acknowledge finanical support by the Erwin Schrödinger Institute, Austrian Academy of Sciences, EPSRC, QIP IRC, Royal Society, Wolfson Foundation, National Research Foundation (Singapore), Ministry of Education (Singapore), Austrian Science Foundation FWF (Project No. P19570-N16 and SFB), European Commission through Project QAP (No. 015846), and FWF Doctoral Program CoQuS.

[1] A. Einstein, B. Podolski, and N. Rosen, Phys. Rev. 47, 777 (1935).

[2] J. S. Bell, Speakable and Unspeakable in Quantum Mechanics (Cambridge University Press, Cambridge, 1987).

[3] M. A. Nielsen and I. L. Chuang, Quantum Computation and Quantum Information (Cambridge University Press, 2000).

[4] K. Banaszek and K. Wódkiewicz, Phys. Rev. A 58, 4345 (1998).

[5] E. P. Wigner, Phys. Rev. 40, 749 (1932).

[6] K. Banaszek and K. Wódkiewicz, Acta Phys. Slov. 49, 491 (1999).

[7] W. J. Munro, Phys. Rev. A 59, 4197 (1999).

[8] Z.-B. Chen et al., Phys. Rev. Lett. 88, 040406 (2002).

[9] E. R. Loubenets, J. Phys. A: Math. Theor. 41, 445303 (2008); E. R. Loubenets, J. Phys. A: Math. Theor. 41, 445304 (2008).

[10] R. Schack and C. M. Caves, Phys. Rev. A 60, 4354 (1999).

[11] N. Linden and S. Popescu, Phys. Rev. Lett. 87, 047901 (2001); J. Kofler and Č. Brukner, Phys. Rev. Lett. 101, 090403 (2008).

[12] J. F. Clauser et al., Phys. Rev. Lett. 23, 880 (1969).

[13] R. J. Glauber, Phys. Rev. Lett. 10, 84 (1963).

[14] S. M. Barnett and P. M. Radmore, Methods in Theoretical Quantum Optics (Clarendon Press, Oxford, 1997). 